\title{
The Global Cyclicity Index of Benzenoid Chains
}

\author{
Yujun Yang, ${ }^{1,2}$ Yan Wang, ${ }^{1}$ and $\mathrm{Yi} \mathrm{Li}^{1}$ \\ ${ }^{1}$ School of Mathematics and Information Science, Yantai University, Yantai, Shandong 264005, China \\ ${ }^{2}$ School of Mathematics, Shandong University, Jinan, Shandong 250010, China
}

Correspondence should be addressed to Yujun Yang; yangyj@yahoo.com

Received 28 May 2013; Accepted 20 September 2013

Academic Editor: Arturo Espinosa

Copyright ( 2013 Yujun Yang et al. This is an open access article distributed under the Creative Commons Attribution License, which permits unrestricted use, distribution, and reproduction in any medium, provided the original work is properly cited.

\begin{abstract}
The resistance distance $\Omega_{G}(i, j)$ between vertices $i$ and $j$ of a connected (molecular) graph $G$ is computed as the effective resistance between nodes $i$ and $j$ in the corresponding network constructed from $G$ by replacing each edge of $G$ with a unit resistor. The conductance excess between any $i$ and $j$ of $G$ is the difference between $1 / \Omega_{G}(i, j)$ and the reciprocal of the distance between $i$ and $j$. The global cyclicity index of $G$ is defined as the sum of conductance excesses between all pairs of adjacent vertices. In this paper, by computing resistance distances between pairs of adjacent vertices in linear polyacenes, an explicit formula for the global cyclicity index of a benzenoid chain is obtained in terms of its number of hexagons.
\end{abstract}

\section{Introduction}

As the number of possible chemical compounds is so big, their huge part will never be experimentally tested. For this reason, there is a need for mathematical modeling and analysis of certain classes of compounds. To this end, many topological indices are defined and applied in the modeling of chemical and pharmacological properties of molecules. In the present work, we will take a new molecular cyclicity measure into consideration.

There are different possible measures of "cyclicity" of a molecular graph $G=(V(G), E(G))$. One simple such traditional fundamental measure is the cyclomatic number $\mu(G)$ (also called the first Betti number, the nullity, or the cycle rank) which is defined for a connected graph $G$ with $n$ vertices and $m$ edges as

$$
\mu(G)=m-n+1 .
$$

Motivated from electrical network theory, Klein and Ivanciuc proposed a new cyclicity measure. This new cyclicity measure is established on the basis of the novel concept of resistance distance [1-7]. As an intrinsic graph metric, the resistance distance $\Omega_{G}(i, j)$ between vertices $i$ and $j$ of a connected (molecular) graph $G$ is computed as the effective resistance between nodes $i$ and $j$ in the corresponding network constructed from $G$ by replacing each edge of $G$ with a unit resistor. Comparing to the traditional (shortest path) distance $d_{G}(i, j)$ between $i$ and $j$, it is well known that $\Omega_{G}(i, j)$ equals the length $d_{G}(i, j)$ of the shortest path between $i$ and $j$ if there is a unique single path between $i$ and $j$, while if there is more than one path, then $\Omega_{G}(i, j)$ is strictly less than $d_{G}(i, j)$. Thence, the conductance excess $\sigma_{G}(i, j)-1 / d_{G}(i, j)$ indicates in some manner the presence of cyclicity in the portion of the graph interconnecting $i$ and $j$, where $\sigma_{G}(i, j)=1 / \Omega_{G}(i, j)$ is known as the effective conductance between $i$ and $j$. To measure the cyclicity of a graph $G$, Klein and Ivanciuc [8] proposed the global cyclicity index $C(G)$ as

$$
C(G)=\sum_{i \sim j}\left[\sigma_{G}(i, j)-\frac{1}{d_{G}(i, j)}\right]
$$

where $i \sim j$ means that $i$ and $j$ are adjacent and the sum is over all edges of $G$. Since $d_{G}(i, j)=1$ for $i \sim j, C(G)$ can also be written as

$$
C(G)=\sum_{i \sim j}\left[\sigma_{G}(i, j)-1\right]=\sum_{i \sim j} \sigma_{G}(i, j)-|E(G)| .
$$

As a new measure of cyclicity of graphs, the global cyclicity index has less degeneracy than the standard cyclomatic number and has some intuitively appealing features. Since the idea of cyclicity is related to measures of connectivity or complexity [9] and characterization of "cyclicity" is an 


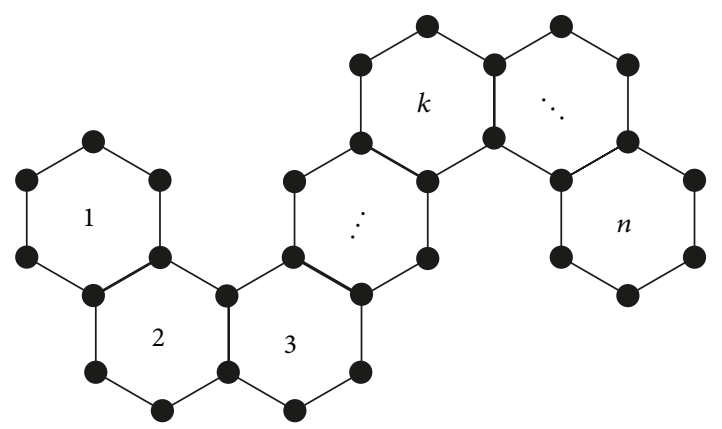

(a)

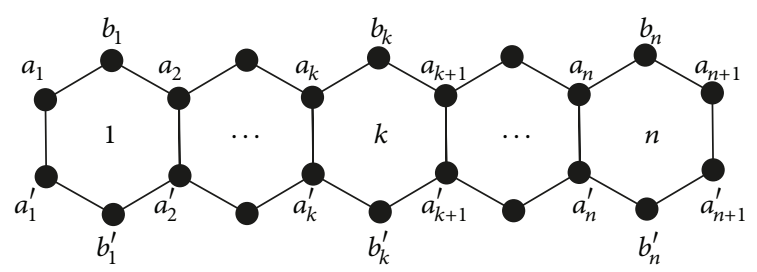

(b)

Figure 1: A benzenoid chain with $n$ hexagons (a) and the linear polyacene $L_{n}$ (b).

aspect of key importance in the study of molecular graphs $[10,11]$, it is worth studying the global cyclicity index from both mathematical and chemical points of view.

A benzenoid system is a 1-connected collection of congruent regular hexagons arranged in the plane in such a way that any two hexagons having a common point intersect in a whole edge. The vertices lying on the border of the unbounded face of a benzenoid system are called external; other vertices, if present, are called internal. A benzenoid system without internal vertices is called catacondensed. If no hexagon in a catacondensed benzenoid is adjacent to three other hexagons, we say that the benzenoid is a benzenoid chain. If a benzenoid chain has no turn hexagons, then it is called a linear polyacene.

In [8], Klein and Ivanciuc established a number of theorems for the global cyclicity index of graphs (even not connected). In [12], one of the present authors obtained bounds for the global cyclicity index of fullerene graphs. In [13], one of the present authors also obtained some further results on the global cyclicity number, including the strictly monotone increasing property, some lower and upper bounds, and some Nordhaus-Gaddum-type results, a relationship between $C(G)$ and the cyclomatic number $\mu(G)$. In this paper, by computing resistance distances between pairs of adjacent vertices in linear polyacenes, an explicit formula for the global cyclicity index of benzenoid chains is obtained in terms of the number of hexagons.

\section{Results}

2.1. Resistance Distances between Adjacent Vertices in Linear Polyacenes. In this subsection, we will compute resistance distances between pairs of adjacent vertices in linear polyacenes. Denote by $L_{n}$ the linear polyacene with $n$ hexagons. For convenience, we label the vertices of $L_{n}$ as depicted in Figure 1.

To compute resistance distances between pairs of adjacent vertices in $L_{n}$, we need to employ the classical result of com puting resistance distances in terms of spanning trees and spanning bi-trees. A spanning tree (resp., forest) of a connected graph $G$ is a subgraph that contains all the vertices and is a tree (resp., forest). A spanning bi-tree of a connected graph is defined as a spanning forest of the graph with exactly two components. A spanning bi-tree is said to separate vertices $i$ and $j$ if the vertices $i$ and $j$ are in distinct components of the bi-tree. For a connected graph $G$ and for any two vertices $i, j \in V(G)$, we denote by $t(G)$ and $t(G ; i, j)$ the number of spanning trees of $G$ and the number of spanning bi-trees of $G$ separating $i$ and $j$, respectively. Then resistance distances can be computed as given in the following Lemma.

Lemma 1 (see [14]). Let $G$ be a connected graph. Then the resistance distance between any two vertices $i$ and $j$ in $G$ can be computed as

$$
\Omega_{G}(i, j)=\frac{t(G ; i, j)}{t(G)} .
$$

Now suppose that $i$ and $j$ are adjacent in $G$, and let $e$ be an edge connecting them. If $T$ is a spanning tree that contains $e$, then by the deletion of $e$ from $T$ we could obtain a spanning bi-tree $F$ separating $i$ and $j$. Conversely, if $F$ is a spanning bi-tree separating $i$ and $j$, then by adding an new edge $e$ connecting $i$ and $j$ to $F$ we could obtain a spanning tree of $G$. So, the number of spanning trees containing $e$ is equal to the number of spanning bi-trees separating $i$ and $j$. While, on the other hand, the number of spanning trees of $G$ that contain $e$ is equal to the number of spanning trees of the graph $G / e$, where $G / e$ denotes the graph obtained from $G$ by contracting $e$, that is, deleting $e$ and then identifying two end vertices of $e$. Thus, as a consequence of Lemma 1, we have the following result.

Lemma 2. Let $G$ be a connected graph with $e=i j \in E(G)$. Then

$$
\Omega_{G}(i, j)=\frac{t(G / e)}{t(G)} .
$$

In the enumeration of spanning trees of graphs, there is a famous recursion formula, known as the deletion-contraction recurrence. As stated in the following lemma, the recursion formula is applicable to multiple graphs (i.e., graphs with multiple edges).

Lemma 3. Let $G$ be a multiple graph, and let e be an edge of G. Then

$$
t(G)=t(G-e)+t\left(\frac{G}{e}\right)
$$


where $G-e$ denotes the graph obtained from $G$ by the deletion ofe.

Combining Lemmas 2 and 3, we readily have Lemma 4.

Lemma 4. Let $G$ be a connected graph with $e=i j$ being an edge of $G$. Then

$$
\Omega_{G}(i, j)=\frac{t(G)-t(G-e)}{t(G)} .
$$

For computing resistance distances between adjacent vertices in $L_{n}$, the number of spanning trees of $L_{n}$ plays an essential rule.

Lemma 5 (see [15-17]). Consider

$$
t\left(L_{n}\right)=\frac{\sqrt{2}}{8}\left[(3+2 \sqrt{2})^{n+1}-(3-2 \sqrt{2})^{n+1}\right] .
$$

Now we are arriving at Theorem 6.

Theorem 6. Resistance distances between pairs of adjacent vertices of a linear polyacene could be computed as follows.

For $1 \leq k \leq n$,

$$
\begin{aligned}
& \Omega_{L_{n}}\left(a_{k}, b_{k}\right) \\
& =\Omega_{L_{n}}\left(b_{k}, a_{k+1}\right)=\Omega_{L_{n}}\left(a_{k}^{\prime}, b_{k}^{\prime}\right)=\Omega_{L_{n}}\left(b_{k}^{\prime}, a_{k+1}^{\prime}\right) \\
& =\left(\left[1-\frac{\sqrt{2}}{8}+\frac{\sqrt{2}}{8}(3-2 \sqrt{2})^{2 k}\right](3+2 \sqrt{2})^{n+1}\right. \\
& \left.\quad-\left[1+\frac{\sqrt{2}}{8}-\frac{\sqrt{2}}{8}(3+2 \sqrt{2})^{2 k}\right](3-2 \sqrt{2})^{n+1}\right) \\
& \quad \times\left((3+2 \sqrt{2})^{n+1}-(3-2 \sqrt{2})^{n+1}\right)^{-1},
\end{aligned}
$$

for $1 \leq k \leq n+1$,

$$
\begin{aligned}
\Omega_{L_{n}}\left(a_{k}, a_{k}^{\prime}\right) & \\
= & \frac{\sqrt{2}}{2}\left(\left[1+(3-2 \sqrt{2})^{2 k-1}\right](3+2 \sqrt{2})^{n+1}\right. \\
& \left.+\left[1+(3+2 \sqrt{2})^{2 k-1}\right](3-2 \sqrt{2})^{n+1}\right) \\
& \times\left((3+2 \sqrt{2})^{n+1}-(3-2 \sqrt{2})^{n+1}\right)^{-1} .
\end{aligned}
$$

Proof. We first compute resistance distances between adjacent degree two and degree three vertices. For $1 \leq k \leq n$, by the structure of $L_{n}$, we could see that

$$
\Omega_{L_{n}}\left(a_{k}, b_{k}\right)=\Omega_{L_{n}}\left(b_{k}, a_{k+1}\right)=\Omega_{L_{n}}\left(a_{k}^{\prime}, b_{k}^{\prime}\right)=\Omega_{L_{n}}\left(b_{k}^{\prime}, a_{k+1}^{\prime}\right) .
$$

By Lemma 4, we have

$$
\Omega_{L_{n}}\left(a_{k}, b_{k}\right)=\frac{t\left(L_{n}\right)-t\left(L_{n}-a_{k} b_{k}\right)}{t\left(L_{n}\right)} .
$$

Notice that the number of spanning trees of the graph $L_{n}-$ $a_{k} b_{k}$ is equal to the product of the number of spanning trees of $L_{k-1}$ and the number of spanning trees of $L_{n-k}$ (here the number of spanning trees of $L_{0}$ is regarded as 1 , and the formula in Lemma 5 is also applicable to $L_{0}$ ); that is,

$$
t\left(L_{n}-a_{k} b_{k}\right)=t\left(L_{k-1}\right) t\left(L_{n-k}\right) .
$$

Hence,

$$
\Omega_{L_{n}}\left(a_{k}, b_{k}\right)=\frac{t\left(L_{n}\right)-t\left(L_{k-1}\right) t\left(L_{n-k}\right)}{t\left(L_{n}\right)} .
$$

Substituting (8) into the above equality, we could obtain (9). It should be mentioned that to obtain (9), one should notice that $3+2 \sqrt{2}$ is the reciprocal of $3-2 \sqrt{2}$.

Now, we compute resistance distances between $\left\{a_{k}\right.$, $\left.a_{k}^{\prime}\right\}(1 \leq k \leq n+1)$. Denote by $S_{n}$ the graph obtained from $L_{n}$ by contracting the edge $a_{1} b_{1}$; that is, $S_{n}=L_{n} / a_{1} b_{1}$. Noticing that the number of spanning trees of $L_{n}-a_{1} b_{1}$ is equal to the number of spanning trees of $L_{n-1}$, by Lemma 3, simple calculation leads to

$$
\begin{aligned}
t\left(S_{n}\right) & =t\left(L_{n}\right)-t\left(L_{n}-a_{1} b_{1}\right)=t\left(L_{n}\right)-t\left(L_{n-1}\right) \\
& =\frac{\sqrt{2}}{4}\left[(\sqrt{2}+1)^{2 n+1}+(\sqrt{2}-1)^{2 n+1}\right] .
\end{aligned}
$$

If we regard the number of spanning trees of $S_{0}$ as 1 , then (15) also holds for $n=0$. Let $L_{n} / a_{k} a_{k}^{\prime}$ be the graph obtained from $L_{n}$ by contracting the edge $a_{k} a_{k}^{\prime}$. Then it is not hard to observe that

$$
t\left(\frac{L_{n}}{a_{k} a_{k}^{\prime}}\right)=t\left(S_{k-1}\right) t\left(S_{n-k+1}\right) .
$$

Hence, by Lemma 2, for $1 \leq k \leq n+1$,

$$
\Omega_{L_{n}}\left(a_{k}, a_{k}^{\prime}\right)=\frac{t\left(L_{n} / a_{k} a_{k}^{\prime}\right)}{t\left(L_{n}\right)}=\frac{t\left(S_{k-1}\right) t\left(S_{n-k+1}\right)}{t\left(L_{n}\right)} .
$$

Substituting (8) and (15) into the above equality, we could obtain (10).

2.2. The Global Cyclicity Index of Benzenoid Chains. In [8], Klein and Ivanciuc obtained the following result in Lemma 7.

Lemma 7 (see [8]). All benzenoid chains with the same number of hexagons have the same global cyclicity index.

Now we are ready to give the main result of the paper. 
TABle 1

\begin{tabular}{lccccccccc}
\hline$G$ & $K f(G)$ & $G$ & $K f(G)$ & $G$ & $K f(G)$ & $G$ & $K f(G)$ & $G$ & $K f(G)$ \\
\hline$H_{1}$ & 1.2 & $H_{11}$ & 13.927 & $H_{21}$ & 26.669 & $H_{31}$ & 39.39 & $H_{41}$ & 52.122 \\
$H_{2}$ & 2.469 & $H_{12}$ & 15.2 & $H_{22}$ & 27.932 & $H_{32}$ & 40.664 & $H_{42}$ & 53.395 \\
$H_{3}$ & 3.7419 & $H_{13}$ & 16.474 & $H_{23}$ & 29.205 & $H_{33}$ & 41.937 & $H_{43}$ & 54.668 \\
$H_{4}$ & 5.0151 & $H_{14}$ & 17.747 & $H_{24}$ & 30.478 & $H_{34}$ & 43.21 & $H_{44}$ & 55.942 \\
$H_{5}$ & 6.2883 & $H_{15}$ & 19.02 & $H_{25}$ & 31.752 & $H_{35}$ & 44.483 & $H_{45}$ & 57.215 \\
$H_{6}$ & 7.5614 & $H_{16}$ & 20.293 & $H_{26}$ & 33.025 & $H_{36}$ & 45.756 & $H_{46}$ & 58.488 \\
$H_{7}$ & 8.8346 & $H_{17}$ & 21.566 & $H_{27}$ & 34.298 & $H_{37}$ & 47.029 & $H_{47}$ & 59.761 \\
$H_{8}$ & 10.108 & $H_{18}$ & 22.839 & $H_{28}$ & 35.571 & $H_{38}$ & 48.303 & $H_{48}$ & 61.034 \\
$H_{9}$ & 11.318 & $H_{19}$ & 24.113 & $H_{29}$ & 36.844 & $H_{39}$ & 49.576 & $H_{49}$ & 62.307 \\
$H_{10}$ & 12.654 & $H_{20}$ & 25.386 & $H_{30}$ & 38.117 & $H_{40}$ & 50.849 & $H_{50}$ & 63.581 \\
\hline
\end{tabular}

Theorem 8. Let $H_{n}$ be a benzenoid chain with $n$ hexagons. Then

$$
\begin{aligned}
C\left(H_{n}\right)=4 \sum_{k=1}^{n}\left(\left[1-(3-2 \sqrt{2})^{2 k}\right](3+2 \sqrt{2})^{n+1}\right. & \left.+\left[1+(3+2 \sqrt{2})^{2 k}\right](3-2 \sqrt{2})^{n+1}\right) \\
\times & \left(\left[4 \sqrt{2}-1+(3-2 \sqrt{2})^{2 k}\right](3+2 \sqrt{2})^{n+1}\right. \\
& \left.-\left[4 \sqrt{2}+1-(3+2 \sqrt{2})^{2 k}\right](3-2 \sqrt{2})^{n+1}\right)^{-1} \\
+ & \sum_{k=1}^{n+1}\left(\left[\sqrt{2}-1-(3-2 \sqrt{2})^{2 k-1}\right](3+2 \sqrt{2})^{n+1}\right. \\
& \left.\quad-\left[\sqrt{2}+1+(3+2 \sqrt{2})^{2 k-1}\right](3-2 \sqrt{2})^{n+1}\right) \\
\times\left(\left[1-(3-2 \sqrt{2})^{2 k-1}\right](3+2 \sqrt{2})^{n+1}\right. & \left.+\left[1+(3+2 \sqrt{2})^{2 k-1}\right](3-2 \sqrt{2})^{n+1}\right)^{-1} .
\end{aligned}
$$

Proof. By Lemma 7, we only need to compute the global cyclicity index of $L_{n}$. On one hand, by (9) in Theorem 6, for $1 \leq k \leq n$, we have

$$
\begin{aligned}
& \sigma_{L_{n}}\left(a_{k}, b_{k}\right)-1 \\
& =\sigma_{L_{n}}\left(b_{k}, a_{k+1}\right)-1 \\
& =\sigma_{L_{n}}\left(a_{k}^{\prime}, b_{k}^{\prime}\right)-1=\sigma_{L_{n}}\left(b_{k}^{\prime}, a_{k+1}^{\prime}\right)-1 \\
& =\left((3+2 \sqrt{2})^{n+1}-(3-2 \sqrt{2})^{n+1}\right)
\end{aligned}
$$

$$
\begin{aligned}
& \times\left(\left[1-\frac{\sqrt{2}}{8}+\frac{\sqrt{2}}{8}(3-2 \sqrt{2})^{2 k}\right](3+2 \sqrt{2})^{n+1}\right. \\
& \left.\quad-\left[1+\frac{\sqrt{2}}{8}-\frac{\sqrt{2}}{8}(3+2 \sqrt{2})^{2 k}\right](3-2 \sqrt{2})^{n+1}\right)^{-1}-1 \\
& =\left(\left[1-(3-2 \sqrt{2})^{2 k}\right](3+2 \sqrt{2})^{n+1}\right. \\
& \left.+\left[1+(3+2 \sqrt{2})^{2 k}\right](3-2 \sqrt{2})^{n+1}\right) \\
& \times\left(\left[4 \sqrt{2}-1+(3-2 \sqrt{2})^{2 k}\right](3+2 \sqrt{2})^{n+1}\right. \\
& \left.\quad-\left[4 \sqrt{2}+1-(3+2 \sqrt{2})^{2 k}\right](3-2 \sqrt{2})^{n+1}\right)^{-1} .
\end{aligned}
$$

On the other hand, by (10), for $1 \leq k \leq n+1$, we have

$$
\begin{aligned}
\sigma_{L_{n}}\left(a_{k}, a_{k}^{\prime}\right)-1 \\
=\left(\sqrt{2}\left[(3+2 \sqrt{2})^{n+1}-(3-2 \sqrt{2})^{n+1}\right]\right) \\
\times\left(\left[1+(3-2 \sqrt{2})^{2 k-1}\right](3+2 \sqrt{2})^{n+1}\right. \\
\left.\quad+\left[1+(3+2 \sqrt{2})^{2 k-1}\right](3-2 \sqrt{2})^{n+1}\right)^{-1}-1 \\
=\left(\left[\sqrt{2}-1-(3-2 \sqrt{2})^{2 k-1}\right](3+2 \sqrt{2})^{n+1}\right. \\
\left.\quad-\left[\sqrt{2}+1+(3+2 \sqrt{2})^{2 k-1}\right](3-2 \sqrt{2})^{n+1}\right) \\
\times\left(\left[1-(3-2 \sqrt{2})^{2 k-1}\right](3+2 \sqrt{2})^{n+1}\right. \\
\left.\quad+\left[1+(3+2 \sqrt{2})^{2 k-1}\right](3-2 \sqrt{2})^{n+1}\right)^{-1} .
\end{aligned}
$$


By the definition of the global cyclicity index, it follows that

$$
\begin{aligned}
C(G)=\sum_{k=1}^{n}\left[\sigma_{L_{n}}\left(a_{k}, b_{k}\right)-1+\sigma_{L_{n}}\left(b_{k}, a_{k+1}\right)\right. \\
\left.\quad-1+\sigma_{L_{n}}\left(a_{k}^{\prime}, b_{k}^{\prime}\right)-1+\sigma_{L_{n}}\left(b_{k}^{\prime}, a_{k+1}^{\prime}\right)-1\right] \\
+\sum_{k=1}^{n+1}\left[\sigma_{L_{n}}\left(a_{k}, a_{k}^{\prime}\right)-1\right] \\
=4 \sum_{k=1}^{n}\left[\sigma_{L_{n}}\left(a_{k}, b_{k}\right)-1\right]+\sum_{k=1}^{n+1}\left[\sigma_{L_{n}}\left(a_{k}, a_{k}^{\prime}\right)-1\right] .
\end{aligned}
$$

Then (18) could be obtained by substituting (19) and (20) into (21).

As numerical results, global cyclicity indices of benzenoid chains from $H_{1}$ to $H_{50}$ are listed in Table 1.

\section{Acknowledgments}

The authors acknowledge the support of the National Natural Science Foundation of China under Grant no. 11371307. Y. Yang acknowledges the support of the National Natural Science Foundation of China under Grant no. 11201404, China Postdoctoral Science Foundation under Grant nos. 2012M521318 and 2013T60662, Special Funds for Postdoctoral Innovative Projects of Shandong Province under Grant no. 201203056, and Shandong Province Higher Educational Science and Technology Program through Grant J12LI05. Y. Wang and Y. Li acknowledge the support of the Natural Science Foundation of Shandong Province under Grant no. ZR2011AM005.

\section{References}

[1] G. E. Sharpe, "Solution of the $(m+1)$-terminal resistive network problem by means of metric geometry," in Proceedings of the 1st Asilomar Conference on Circuits and Systems, pp. 319-328, Pacific Grove, Calif, USA, November 1967.

[2] G. E. Sharpe, "Theorem on resistive networks," Electronics Letters, vol. 3, no. 10, pp. 444-445, 1967.

[3] G. E. Sharpe, "Violation of the 2-triple property by resistive networks," Electronics Letters, vol. 3, no. 12, pp. 543-544, 1967.

[4] A. D. Gvishiani and V. A. Gurvich, "Metric and ultrametric spaces of resistances," Russian Mathematical Surveys, vol. 42, no. 2, pp. 235-236, 1987.

[5] V. Gurvich, "Metric and ultrametric spaces of resistances," Discrete Applied Mathematics, vol. 158, no. 14, pp. 1496-1505, 2010.

[6] D. J. Klein and M. Randić, "Resistance distance," Journal of Mathematical Chemistry, vol. 12, no. 1, pp. 81-95, 1993.

[7] P. Y. Chebotarev and E. V. Shamis, "The forest metrics of a graph and their properties," Automation and Remote Control, vol. 61, no. 8, pp. 1364-1373, 2000.

[8] D. J. Klein and O. Ivanciuc, "Graph cyclicity, excess conductance, and resistance deficit," Journal of Mathematical Chemistry, vol. 30, no. 3, pp. 271-287, 2001.
[9] W. Tutte, Connectivity in Graphs, University of Toronto Press, Toronto, Canada, 1966.

[10] D. Bonchev, O. Mekenyan, and N. Trinajstić, "Topological characterization of cyclic structures," International Journal of Quantum Chemistry, vol. 17, no. 5, pp. 845-893, 1980.

[11] D. Bonchev, A. T. Balaban, X. Liu, and D. J. Klein, "Molecular cyclicity and centricity of polycyclic graphs. I. Cyclicity based on resistance distances or reciprocal distances," International Journal of Quantum Chemistry, vol. 50, pp. 1-20, 1994.

[12] Y. Yang, "Resistance distances and the global cyclicity index of fullerene graphs," Digest Journal of Nanomaterials and Biostructures, vol. 7, pp. 593-598, 2012.

[13] Y. Yang, "On a new cyclicity measure of graphs-the global cyclicity index," Discrete Applied Mathematics. Accepted.

[14] S. Seshu and M. B. Reed, Linear Graphs and Electrical Networks, Addison-Wesley, Reading, Mass, USA, 1961.

[15] D. M. Cvetković and I. Gutman, "A new spectral method for determining the number of spanning trees," Publications de l'Institut Mathématique, vol. 29, no. 43, pp. 49-52, 1981.

[16] I. Gutman and R. B. Mallion, "On spanning trees in catacondensed molecules," Zeitschrift für Naturforschung A, vol. 48, no. 10, pp. 1026-1030, 1993.

[17] E. J. Farrell, M. L. Gargano, and L. V. Quintas, "Spanning trees in linear polygonal chains," Bulletin of the ICA, vol. 39, pp. 67-74, 2003. 

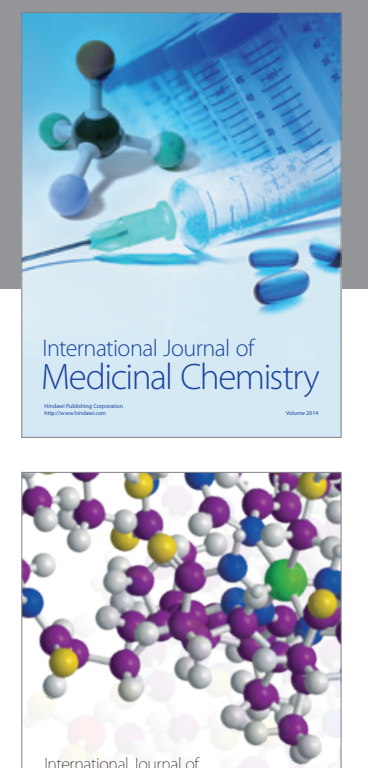

\section{Carbohydrate} Chemistry

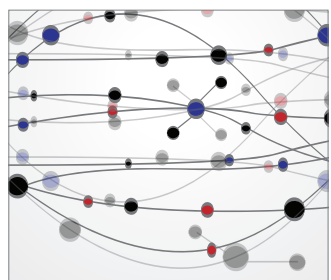

The Scientific World Journal
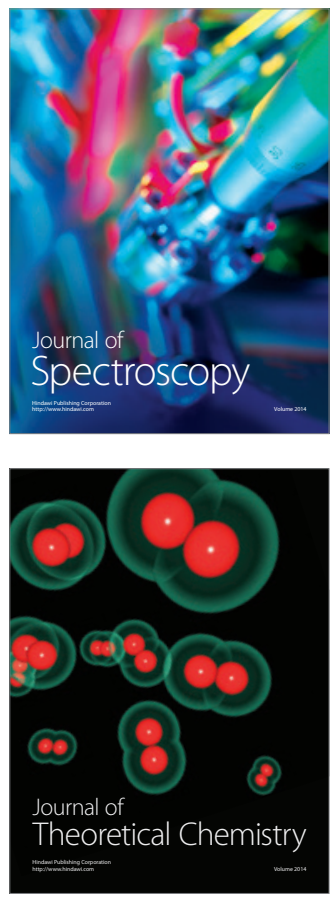
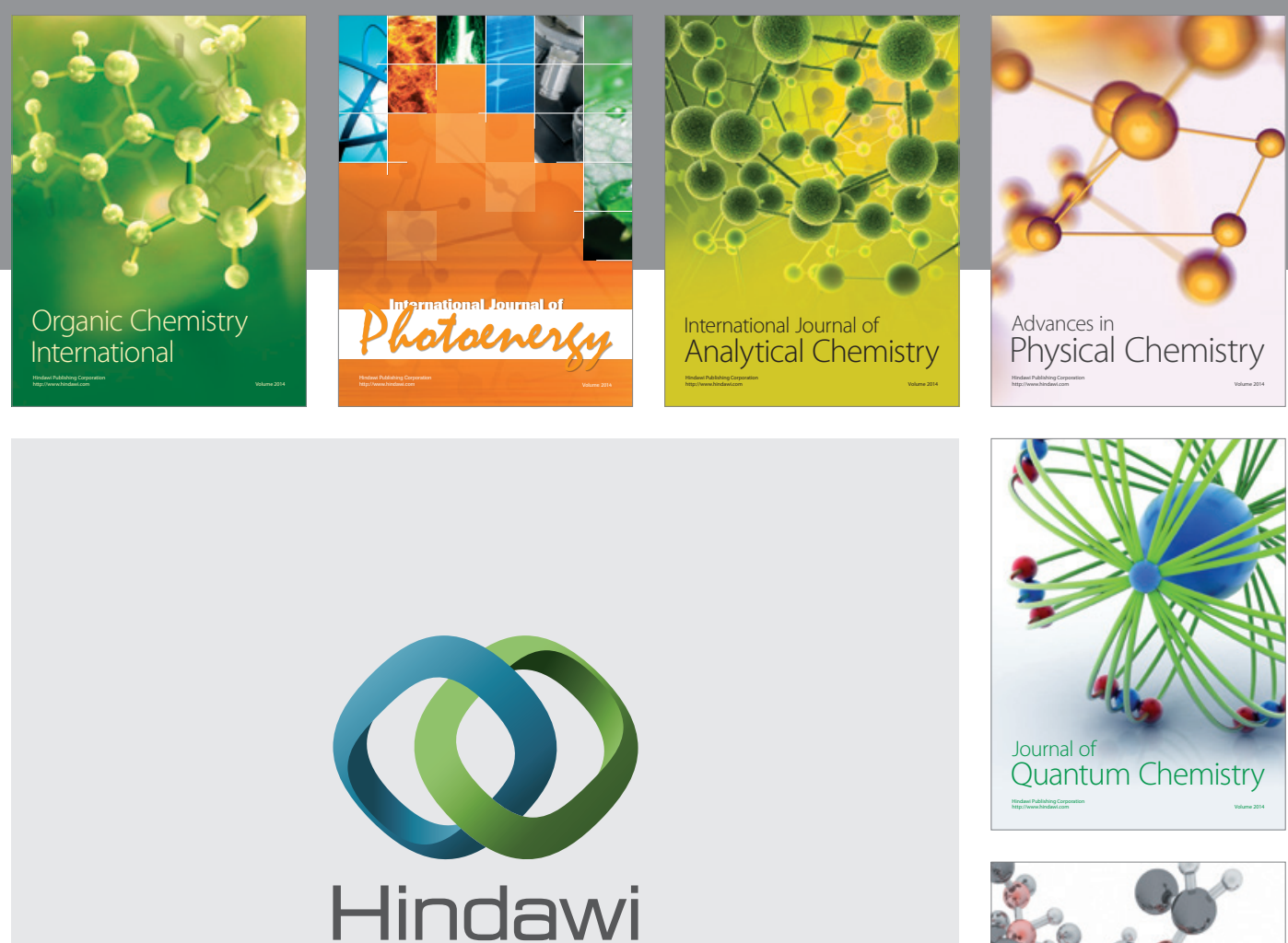

Submit your manuscripts at

http://www.hindawi.com

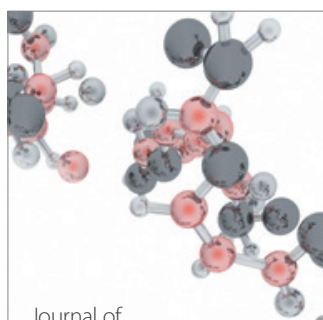

Analytical Methods

in Chemistry

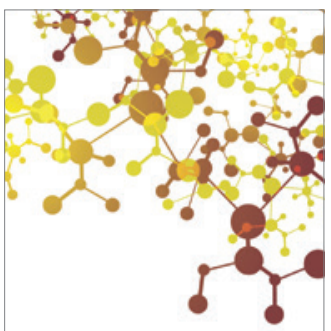

Journal of

Applied Chemistry

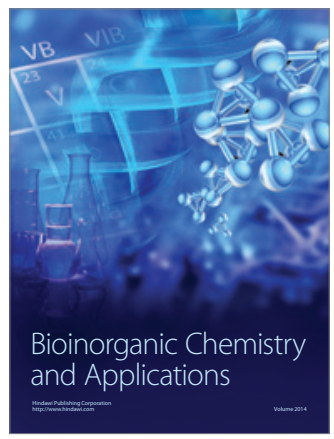

Inorganic Chemistry
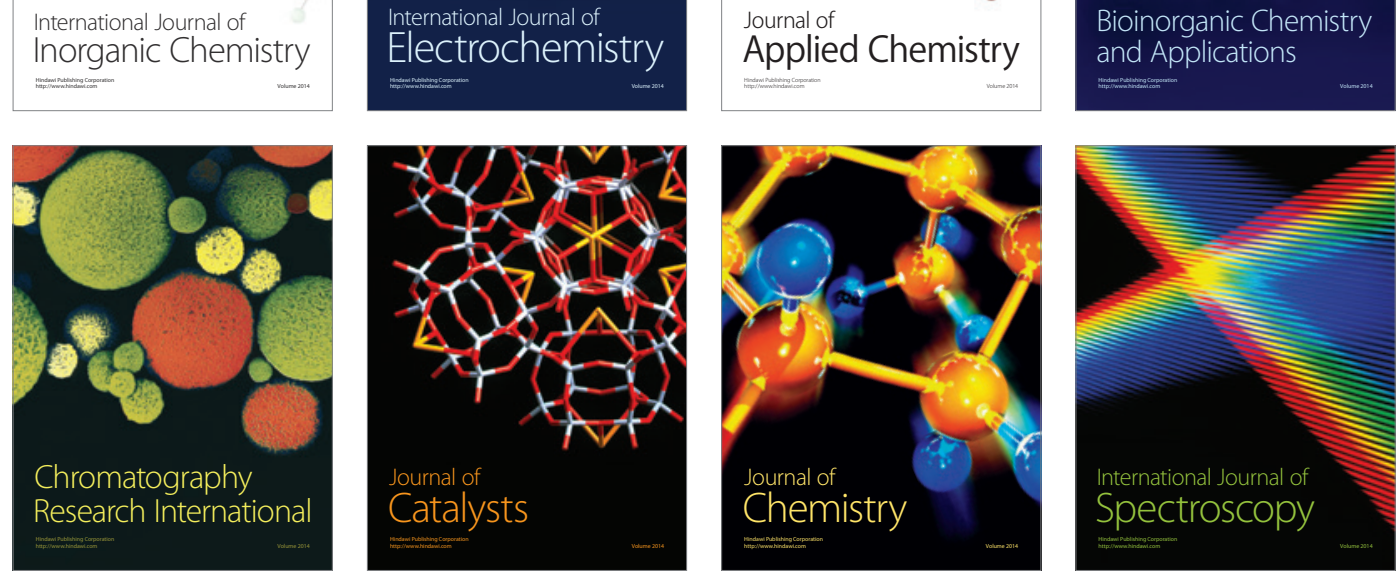